\section{A possible practical application of heavy quark physics}

A POSSIBLE method for storing very large amounts of useable energy within small volumes is described here. Our proposal is based on the existence of two new, virtually stable, forms of matter that annihilate each other with enormous energy release, but neither of which annihilates ordinary matter (and can thus be stored easily). The existence of these new forms of matter is suggested ${ }^{1,2}$ by the phenomenology of heavy quarks and also by a large class of widely discussed unified field theories.

It has been proposed ${ }^{1,2}$ that the $b$ quark, the constituent of the $\mathrm{T}$ meson ${ }^{3-5}$, might be a colour sextet and as such probably be absolutely stable. The possible absolute stability of the $b$ quark has also been explored from a different viewpoint ${ }^{6-8}$. We assume $e^{1,2,6}$ that the electric charge of the $b$ quark equals $-\frac{1}{3}$ times that of the proton. The $b$ quark carries colour and is therefore confined inside colourless hadrons, that is, observable baryons and mesons. The lightest baryons containing a $b$ (or $\bar{b}$ ) quark are then $B^{+} \equiv b u u, B^{0} \equiv b u d$, and the lightest mesons are $M^{+} \equiv \bar{b} u$ and its antiparticle $M^{-} \equiv b \bar{u}$. Being each other's antiparticles $M^{-}$and $M^{+}$have the same mass: $m_{M^{+}}=m_{M}$, while the $B^{+}$and $B^{0}$ masses differ: $m_{B^{+}} \neq m_{B^{0}}$. For $m_{B^{+}}<m_{B^{0}}$ and $\left|m_{B^{+}}-m_{M^{-}}\right|<m_{p}+m_{\pi^{+}} \simeq 1,078 \mathrm{MeV}$ the hadrons $B^{+}$and $M^{ \pm}$ are absolutely stable and $B^{0}$ will undergo $\beta$-decay. Conversely, for $m_{B^{0}}<m_{B^{+}}$and $\left|m_{B^{0}}-m_{M^{-}}\right|<m_{p} \simeq 938 \mathrm{MeV}, B^{\circ}$ and $M^{ \pm}$are absolutely stable. Standard quark model reasoning strongly suggests the validity of these meson-baryon mass-difference inequalities. Thus the meson $M^{+}$and one of the baryons, $B^{+}$or $B^{0}$, are expected to be stable. Whichever baryon is stable $\left(B^{+}\right.$or $B^{0}$ ) the following three observations apply: (1) neither $M^{+}$nor $B^{+}, B^{0}$ contain $\bar{u}$ or $\bar{d}$ antiquarks and therefore do not annihilate ordinary matter; (2) the positively charged hadrons $M^{+}$and $B^{+}$ can form hydrogenoid atoms, which we denote $M\left(M^{+} e^{-}\right)$and $\mathscr{B}\left(B^{+} e^{-}\right)$, with ordinary negative electrons. These electrically neutral atoms are stable and, as they contain electrons rather than positrons, they do not annihilate ordinary matter either. Hence they may participate in ordinary chemical reactions; (3) $M^{+}$contains one $\vec{b}$ but $B^{+}, B^{0}$ each contain one $b$ quark, so that $M^{+}$can annihilate with both $B^{+}$and $B^{0}$.

Several intriguing new possibilities arise. The most attractive one is to assume first stable $M^{+}$and $B^{+}$. The resulting hydrogenoid gases $\mathscr{M}_{2}$ and $\mathscr{B}_{2}$ may be stored in conventional containers and transported separately, or the compound $\mathcal{M} \mathscr{B}$ may be formed and transported itself. Other more complex chemical compounds would certainly exist. These two new forms of matter $\mathcal{M}$ and $\mathscr{B}$ can be induced to annihilate with considerable release of energy, $\sim 10 \mathrm{GeV}$ per reaction, with typical nuclear cross sections.

The case of a stable $M^{+}$and $B^{\circ}$ is more subtle. Perhaps the $B^{\circ}$ can be attached to a conventional nucleus forming an exotic isotope. For example, if a stable heavy deuterium consisting of $B^{0} p^{+}$exists, then the discussion of the preceding paragraph applies with $\mathscr{B}$ replaced by $B^{0} p^{+} e^{-}$. Other stable or long lived quasi-stable isotopes may exist. If no stable nuclei containing $B^{0}$ exist, then this substance would have to be contained by itself, perhaps using a 'magnetic bottle' with a field of non-uniform gradient exploiting the $B^{0}$ magnetic moment. In this case there would no no Coulomb barrier to overcome in initiating annihilation reactions.

All these possibilities seem to be within the realm of conventional technology. Stable quarks would, therefore, offer the possibility of storing very high (useable) energies within small volumes. The technological possibilities are self-evident. It is, as we have found, an inexpensive device to store antiprotons.

We emphasise that experimentally both the existence and the stability questions for the $B^{0+}$ and $\mathrm{M}^{+}$hadrons are still open. A further problem is to provide sufficient amounts of $B^{0+}$ and $M^{+}$ matter in a reasonable time interval. Nevertheless, the prospect of this new energy source is worth pointing out at this time.
Finally, should the $b$ quark not be absolutely stable or sufficiently long lived, heavier quarks may exist which are. If carrying $-1 / 3$ or $+2 / 3$ proton electric charges, any such quark would be as good in the applications envisaged here.

Since we submitted this note two experimental searches ${ }^{9,10}$ for stable hadrons in the $4-10 \mathrm{GeV} \mathrm{c}^{-2}$ mass range have yielded negative results, thus making the existence of stable $b$-quarkcontaining hadrons highly unlikely. One may have to wait for higher quark species. We have also learned of a paper of $\mathrm{H}$. Fritzsch $^{11}$, in which the results given in refs 1, 2 are independently obtained.

This work was supported in part by the NSF.

\section{PETER G. O. FREUND} CHRISTOPHER T. HILL

The Enrico Fermi Institute, and the Department of Physics, The University of Chicago, Chicago, Illinois 60637

Received 3 July; accepted 2 October 1978

1. Freund, P. G. O. \& Hill, C. T., preprint EFI $78 / 21$ (in the press).

2. Ng, Y. J. \& Tye, S.-H. H., preprint SLAC-PUB-78/30-THY (in the press)

3. Herb, S. W. et al. Phys. Rev. Lett. 39, 252-255 (1977)

4. Berger, C. H. et al. Phys. Lett. 76B, 243-245 (1978)

5. Dardeen, C. W. et al. Phys. Lett. 76B, 246-248 (1978).

6. Cahn, R. Phys. Rev. Lett. 40, 80-83 (1978).

7. Karl, G. Phys. Rev. D14, 2374-2382 (1976)

8. Wilczek, F. \& Zee, Z. Phys. Rev. D16, 860-868 (1977)

9. Cutts, D. et al. Phys. Rev. Lett. 41, 363-367 (1978).

0. Vidal, R. et al. Phys. Lett. 77B, 344-346 (1978).

11. Fritzsch, H. Wuppertal preprint WU-B78-19.

\section{Dyeing increases the friction of synthetic polymer yarns}

WE show here that the friction on metal of poly(ethyleneterephthalate) (PET), nylon-6,6 and polyacrylonitrile (PAN) yarns increases when the yarns are dyed, each with a different dye. This phenomenon is well known in the textile industry, but it has only been investigated quantitatively in one case, the friction of dyed wool on metal ${ }^{1}$. Our results show that it is a general phenomenon, and that it is possible to change the mechanical properties of a polymer by the addition of small molecules which have an affinity for the polymer chains.

The PET, nylon-6,6 and PAN yarns were dyed with a disperse, an acid and a basic dye respectively in various conditions (see legends to Figs 2 and 3 ). The friction of the dyed yarn samples, of the purified but otherwise untreated yarns, and of

Fig. 1 Measurement of yarn friction (see ref. 3 pages 189, 210). Dyed and undyed yarn samples were originally wound on bobbins. The yarn passes round a pulley with a ferromagnetic disk at its centre: the input tension of the yarn is adjusted by moving a permanent magnet nearer or further from the disk. Input tension $T_{1}$ is measured by a tension meter consisting of two small pulleys one of which is on a spring loaded arm. The yarn passes once round a stationary steel rod $1 \mathrm{~cm}$ diameter then over three small pulleys of a second meter: the central pulley is attached to the plates of a condenser, and its capacitance is recorded continuously and calibrated to measure the output tension, $T_{2}$. The yarn is wound on to a bobbin at a constant speed of $12.8 \mathrm{~m} \mathrm{~min}^{-1}$. Pulleys and the rod were cleaned with acetone before each determination.

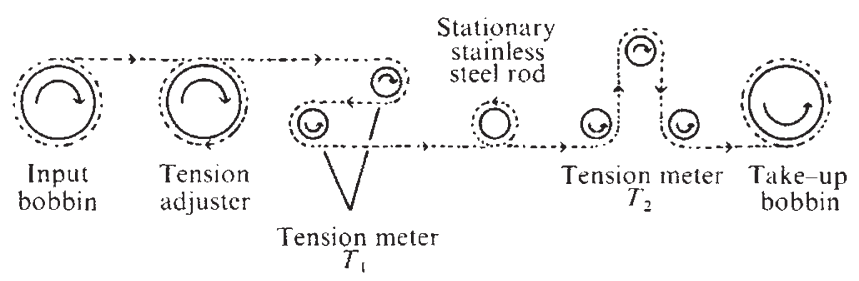

